\title{
Fasciculations, Autonomic Symptoms and Limbic Encephalitis: A Thymoma-Associated Morvan's-Like Syndrome
}

\author{
Feza Deymeer ${ }^{a}$ Sukriye Akca ${ }^{a}$ Gulsen Kocaman ${ }^{a}$ Yesim Parman ${ }^{a}$ Piraye Serdaroglu ${ }^{a}$ \\ Oget Oktem-Tanor ${ }^{\mathrm{a}}$ Oguzhan Coban $^{\mathrm{a}}$ Angela Vincent ${ }^{\mathrm{b}}$

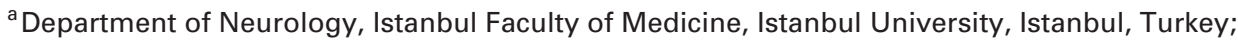 \\ ${ }^{b}$ Neurosciences Group, Weatherall Institute of Molecular Medicine, University of Oxford, John Radcliffe Hospital, \\ Oxford, UK
}

Dear Sir,

Morvan's syndrome or 'chorée fibrillaire de Morvan' consists of peripheral nerve hyperexcitability (PNH), as well as autonomic and central nervous system (CNS) symptoms. With only a limited number of reported cases, the complete spectrum of the CNS symptomatology in Morvan's syndrome has not been well established. These symptoms have been noted to bear a striking similarity to limbic encephalitis (LE) [1-3]; however, there has not been convincing evidence of LE with demonstrable involvement of limbic structures associated with a PNH syndrome except for 2 cases [2, 4].

We present a patient with thymoma and myasthenia gravis who had an episode with fasciculations, autonomic symptoms and memory impairment while in pharmacological remission 9 years after the removal of the thymoma. LE was well defined both by neuropsychological tests and magnetic resonance imaging (MRI) studies.

\section{Case Report}

A 45-year-old man was diagnosed with myasthenia gravis 9 years ago. A malignant lymphoepithelial thymoma was found for which he underwent thymomectomy followed by radiotherapy. He was in pharmacological remission for the last 4 years. He presented with a 20-day history of tingling and burning sensations under the feet, followed by severe pain in the thighs and legs, profuse fasciculations in the lower extremi- ties, tremor in the hands, excessive sweating in the head and hands, impotence, urinary urgency, and insomnia. Physical examination revealed tachycardia and hyperhidrosis. Neurological examination was normal except for fasciculations in the lower extremities and a moderately diminished vibration sense in the toes. There was no postural hypotension. Needle electromyography showed fasciculations in the gastrocnemius muscle. Nerve conduction studies were normal. Autonomic tests revealed decreased RR interval variability and absent sympathetic skin responses. A detailed cardiological evaluation was normal except for sinus tachycardia with a mean heart rate of 110/min during $24 \mathrm{~h}$ Holter monitoring.

One month after the onset of symptoms, there was a sudden onset of memory deficit. Neuropsychological evaluation (Wechsler Memory Scale Digit Span, Logical Memory and Visual Reproduction subtests, backward counting, category fluency test, Wechsler Adult Intelligence Scale-Revised similarities subtest, Stroop test, verbal memory processes test, Luria's alternating sequences) revealed a defect in primary visual memory registration with an attention deficit, but intact primary verbal memory. Contrast-enhanced MRI of the brain (fig. 1a, b) showed high signal and mass effect predominantly in the right hippocampus on fluid-attenuated inversion recovery and $\mathrm{T}_{2-}$ weighted images. Computed tomography of the thorax did not show tumor recurrence.
Prednisolone was increased to $70 \mathrm{mg} /$ day, 1 week after the onset of the CNS symptoms. At the same time, the patient was given $1 \mathrm{~g}$ methylprednisolone intravenously twice a week for a total dose of $5 \mathrm{~g}$. After the onset of treatment, all symptoms gradually resolved within about 6 weeks. As the patient improved, there was a short period of a few days when he complained of brief déjà vu episodes. The electroencephalogram was normal. Cerebrospinal fluid (CSF) was acellular. CSF protein and $\mathrm{IgG}$ index were normal, oligoclonal bands were negative. Pretreatment samples were not available for testing, but voltage-gated potassium channel (VGKC) antibodies were not detected in serum and CSF at this time. The serum was tested on a commercial blot that detects antibodies against $\mathrm{Hu}, \mathrm{Ma}, \mathrm{Yo}$, $\mathrm{Ri}$, collapsing response mediator protein 5 and amphiphysin; all were negative. Sympathetic skin responses returned to normal although the pathological findings in RR interval variability continued. Twenty-fourhour Holter monitoring showed the mean heart rate to be decreased to $90 / \mathrm{min}$. A second neuropsychological evaluation showed complete disappearance of the visual memory registration deficit. A repeat MRI showed marked resolution of the signal abnormality and mass effect (fig. 1c, d).

There was further improvement in the third neuropsychological evaluation. The patient made a complete recovery within 5 months from the onset of the disease at

\section{KARGER}

Fax +4161306 1234 E-Mail karger@karger.ch www.karger.com
(C) 2005 S. Karger AG, Basel 0014-3022/05/0544-0235\$22.00/0

Accessible online at: www.karger.com/ene
Feza Deymeer

Department of Neurology, Istanbul University

Istanbul Faculty of Medicine, Çapa

TR-34390 Istanbul (Turkey)

Tel. +90 21241420 000, Fax +90 212533 8575, E-Mail deymeer@tnn.net 

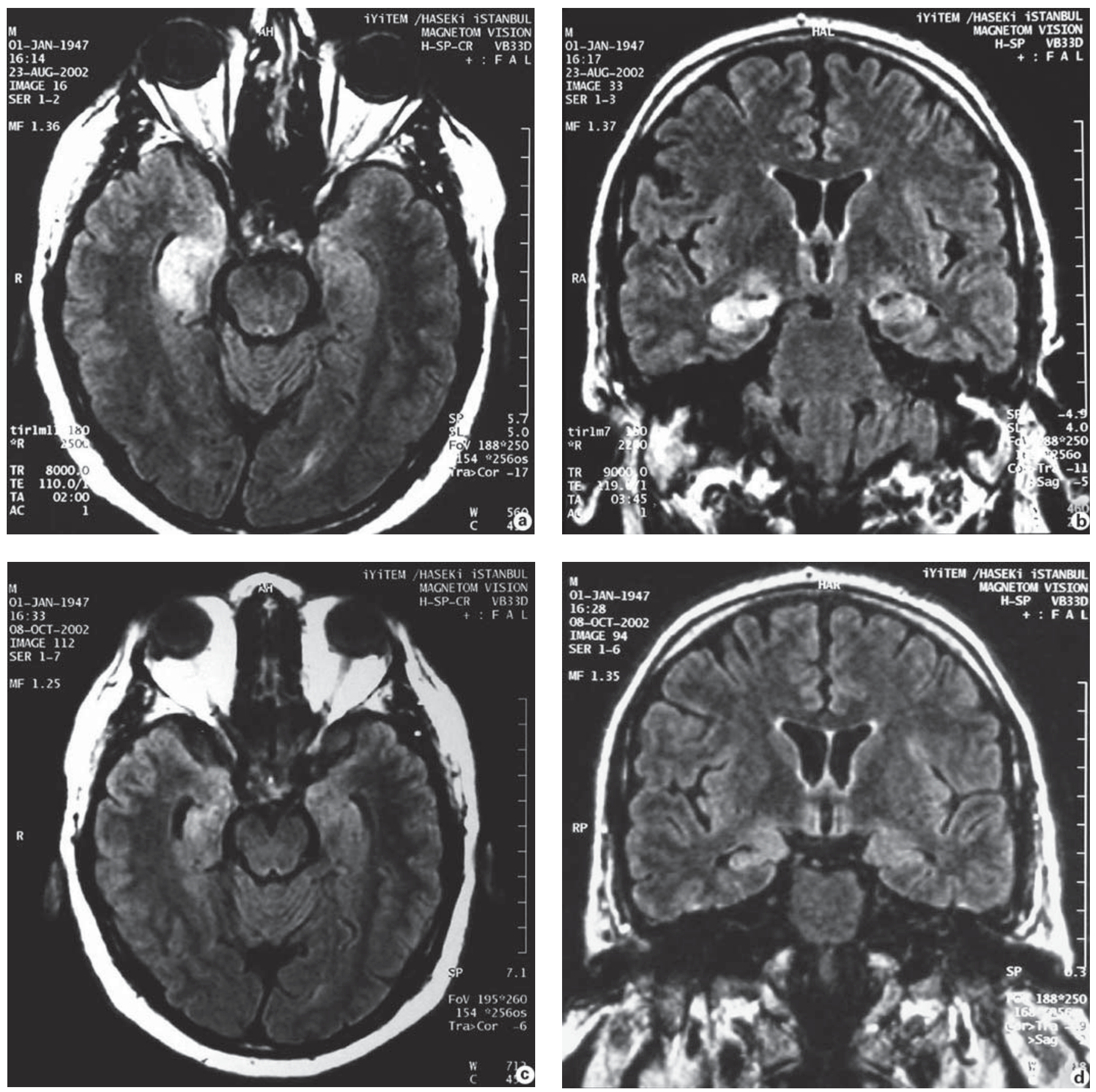

Fig. 1. a, b Transversal and coronal fluid-attenuated inversion recovery MRI shows high signal in the right hippocampus. The right temporal horn of the lateral ventricle is compressed. A subtle signal

abnormality is also present in the left hippocampus. c, d Repeat MRI 2 months later reveals marked resolution of the signal abnormality, reversal of the mass effect, and right hippocampal atrophy.

which time the prednisolone had been decreased to $25 \mathrm{mg} / \mathrm{day}$. Two years later (at the end of 2004), he was still asymptomatic on $10 \mathrm{mg}$ of prednisolone taken every other day.
Discussion

The combination of PNH (clinical and electromyographic fasciculations confined to the lower extremities), autonomic nervous system (hyperhidrosis in the head and hands, sinus tachycardia, urinary urgency, and impotence together with RR interval variability and absent skin responses) and CNS (insomnia and visual memory registration impairment) symptoms/signs in our 
patient most closely resembles Morvan's syndrome.

The CNS symptoms of Morvan's syndrome consist of insomnia, hallucinations, and disorientation, very similar to those seen in LE [1-3]. The presence of oligoclonal bands in the CSF implies immune mediation in both [4-8]. Both entities can be paraneoplastic [1, 2, 5-10] and associated with thymoma [1, 2, 4, 6, 8-10]. Recently, VGKC antibodies, variably reported in Morvan's syndrome [1, 3, 6, 7], have been found in patients with LE [4], strengthening the hypothesis that LE and Morvan's syndrome may be closely connected. The next logical step would be the possibility that the CNS symptoms in the reported cases with Morvan's syndrome are nothing other than manifestations of LE. The major obstacle in the resolution of this issue seems to be the differing degrees of effort put into seeking limbic - particularly hippocampal - structure involvement in the two entities. Careful clinical assessment has demonstrated memory impairment, the hallmark of this syndrome, in cases with LE. Ancillary laboratory tests including MRI and brain biopsy have confirmed temporal lobe involvement [8]. In contrast, memory has not been reported to be tested either clinically or with neuropsychological tests in most Morvan's syndrome cases, perhaps due to agitation and poor cooperation [7]. Of the papers published in the last 30 years [1-7, 9-11], only three mentioned memory impairment $[1,2,4]$, and neuropsychological tests during the symptomatic period were reported in only two [2, 4]. MRI was reported to be performed only in the more recent publications $[2,4,5,7]$. Autopsy was done in three studies $[7,9,11]$.

The situation being as it is, patients with characteristics of Morvan's syndrome who have LE with well-documented memory impairment and good evidence of limbic (hippocampal) involvement are valuable. In the case reported by Evoli et al. [2], PNH (neuromyotonia) and autonomic symptoms started 7 months after the onset of the verbal memory impairment. In a VGKC antibody-positive case reported by Vincent et al. [4], impairment in verbal and visual memory appeared to be cotemporaneous with PNH (neuromyotonia) and autonomic symptoms. In both patients, cranial MRI showed increased signal in the hippocampi. In our patient, a selective memory impairment, a primary registration defect in visual memory, appeared 1 month after the onset of PNH (fasciculations) and auto- nomic symptoms. The visual memory impairment correlated with the right-sided hippocampal lesion as shown on MRI. Later neuropsychological evaluations done after the institution of steroids revealed complete disappearance of the memory impairment in parallel with the improvement in the right hippocampal lesion.

Another interesting feature in our patient was the nature of $\mathrm{PNH}$, consisting of fasciculations in the lower extremities. The electrophysiological manifestations of $\mathrm{PNH}$ have included myokymia and/or neuromyotonia in all cases of Morvan's syndrome. Fasciculation as the sole manifestations of PNH is unusual. Two recent studies suggest that different discharges form a continuum rather than being part of distinct entities [12, 13].

The absence of VGKC antibodies in our patient can have two explanations. Although these antibodies have usually been found to be present, whenever tested, in more recent publications of Morvan's syndrome $[1,3,6,7]$, they were found to be absent in many patients with this diagnosis [12; A. Vincent, unpubl. observations]. An alternative explanation is that they were not detected because they were searched for while the patient had improved (though still symptomatic) on steroids. Indeed, these antibodies can disappear or their titers decrease after improvement $[3,4,6]$.

Plasmapheresis alone [5-7] or in combination with steroids [2], sometimes also with thymectomy and azathioprine [1], has been the most frequently used therapeutic approach in Morvan's syndrome. Although good results were usually obtained, a failed response to steroids and to subsequently added plasmapheresis has been reported [10]. Intravenous immunoglobulin was more effective than plasmapheresis in one report [4], while the reverse was true in another [5]. Gradual spontaneous improvement has also been observed [3]. The dramatic response to high-dose oral prednisolone together with pulse methylprednisolone with almost complete disappearance of the symptoms within a short period in our case should induce consideration of corticosteroids in Morvan's syndrome as well.

This case highlights the difficulties in providing a tight classification of autoimmune PNH and CNS syndromes. More detailed clinical and laboratory examination of the CNS symptoms in future cases with Morvan's syndrome may help to determine whether or not there exists a sound reason for distinguishing these symptoms from those of LE.

\section{References}

1 Lee EK, Maselli RA, Ellis WG, Agius MA: Morvan's fibrillary chorea: a paraneoplastic manifestation of thymoma. J Neurol Neurosurg Psychiatry 1998;65:857-862.

2 Evoli A, Lo Monaco M, Marra R, Lino MM, Batocchi AP, Tonali PA: Multiple paraneoplastic diseases associated with thymoma. Neuromuscul Disord 1999;9:601-603.

3 Barber PA, Anderson NE, Vincent A: Morvan's syndrome associated with voltage-gated $\mathrm{K}^{+}$channel antibodies. Neurology 2000;54: 771-772.

4 Vincent A, Buckley C, Schott JM, Baker I, Dewar BK, Detert N, et al: Potassium channel antibody-associated encephalopathy: a potentially immunotherapy-responsive form of limbic encephalitis. Brain 2004;127:701-712.

5 Madrid A, Gil-Peralta A, Gil-Neciga E, Gonzalez JR, Jarrin S: Morvan's fibrillary chorea: remission after plasmapheresis. J Neurol 1996; 243:350-353.

6 Heidenreich F, Vincent A: Antibodies to ionchannel proteins in thymoma with myasthenia, neuromyotonia, and peripheral neuropathy. Neurology 1998;50:1483-1485.

7 Liguori R, Vincent A, Clover L, Avoni P, Plazzi G, Cortelli P, et al: Morvan's syndrome: peripheral and central nervous system and cardiac involvement with antibodies to voltagegated potassium channels. Brain 2001;124: 2417-2426.

8 Gultekin SH, Rosenfeld MR, Voltz R, Eichen J, Posner JB, Dalmau J: Paraneoplastic limbic encephalitis: neurological symptoms, immunological findings and tumour association in 50 patients. Brain 2000;123:1481-1494.

9 Halbach M, Homberg V, Freund HJ: Neuromuscular, autonomic and central cholinergic hyperactivity associated with thymoma and acetylcholine receptor-binding antibody. J Neurol 1987;234:433-436.

10 Iwasaki Y, Kinoshita M, Ikeda K, Takamiya $\mathrm{K}$, Shiojima T: Concurrence of myasthenia gravis and choreé fibrillaire de Morvan. Eur Arch Psychiatry Neurol Sci 1990;239:335336

11 Fischer-Perroudon C, Trillet M, Mouret J, Tommasi M, Jouvet M, Schott B, et al: Polygraphic and metabolic studies of persistent insomnia with hallucinations. Apropos of an anatomo-clinical study of a case of Morvan's fibrillar chorea. Rev Neurol (Paris) 1974;130: 111-125.

12 Vernino S, Lennon VA: Ion channel and striational antibodies define a continuum of autoimmune neuromuscular hyperexcitability. Muscle Nerve 2002;26:702-707.

13 Hart IK, Maddison P, Newsom-Davis J, Vincent A, Mills KR: Phenotypic variants of autoimmune peripheral nerve hyperexcitability. Brain 2002;125:1887-1895. 